\title{
Detection of Vorticity in Flow Fields
}

\author{
A. M. L. Kappers, A. J. van Doorn, and J. J. Koenderink \\ Utrecht Biophysics Research Institute, The Netherlands
}

\begin{abstract}
Visual detection of vorticity in the presence of a translational component is studied. Stimuli are sparse random dots patterns. The center of rotation is under all circumstances located outside the stimulus window. Task of the subjects is to discriminate between clockwise and anti-clockwise rotation. As expected, vorticity detection thresholds depend critically on the average translational speed. Other important parameters are the life time of the dots and the number of dots.
\end{abstract}

\section{Introduction}

Optical flow fields are of fundamental importance for optically guided behavior such as navigation, orientation, and the maintenance of posture (Gibson, 1950). The relevant information is mainly contained in the structure of the flow field, and not in, for instance, the average direction or speed of the flow. For such tasks in which the global structure of the environment is of principal importance (example: the floor or groundplane) it is mainly the first order (linear) structure that contains the information (Koenderink \& Van Doorn, 1984; Warren, Morris, \& Kalish, 1988). For such tasks as shape perception and recognition higher order structure is involved. The first order structure can be decomposed into elementary components such as translation, rotation, dilation, and pure shear.

The experiments described here are part of a systematic psychophysical investigation on the detection of structure in optic flow fields. We study the detection of certain elementary components in their dependency as the magnitude of another one. In this paper we address the detection of vorticity (clockwise or anti-clockwise rotation) in its dependency on the average translational speed.

\section{Methods}

Apparatus. The stimuli are generated on an Atari 1040 ST computer. An Atari SM 125 high resolution $70 \mathrm{~Hz}$ white phosphor P4 monochrome monitor is used (luminance $71 \mathrm{~cd} / \mathrm{m}^{2}$ ). The monitor dimensions are $13.6 \times 21.7 \mathrm{~cm}(400 \times 640$ pixels), and the monitor is viewed monocularly with a natural pupil from a distance of $34 \mathrm{~cm}$. Thus the field of view is $22.6 \times 35.4^{\circ}$ of visual angle ( 1 pixel $=3.4$ arcmin.). 
Stimulus. The spatial configuration of the dots is based on a regular hexagonal grid. The total number of dots $(4,8,16,24,32,48$, or 64$)$ decides the grid spacing. The regular grid is slightly perturbed with a two-dimensional Gaussian perturbation vector of spread $1 / 8$ of the grid spacing. We generate moving patterns by stroboscopically presenting sequences of frames. In consecutive frames all individual dots change position in a deterministic way. The total presentation time, which is determined by the number of frames, is kept constant (16 frames, $228 \mathrm{~ms}$ ). The lifetime of the dots is an experimental parameter and can be $2,3,4$, or 16 (maximum) frames. The diameter of the stimulus is always 380 pixels $\left(21.5^{\circ}\right)$. A speed of 1 pixel/frame corresponds to $3.95^{\circ} / \mathrm{s}$.

The deterministic component of the stimulus movement consists of a rotation (vorticity), either clockwise or anti-clockwise. The center of rotation lies outside the visible region on a horizontal axis through the center of the stimulus. The average translation inside the stimulus window is directed downwards. In Figure 1 an example of the stimulus is shown. Here the 16 consecutive frames are superimposed.
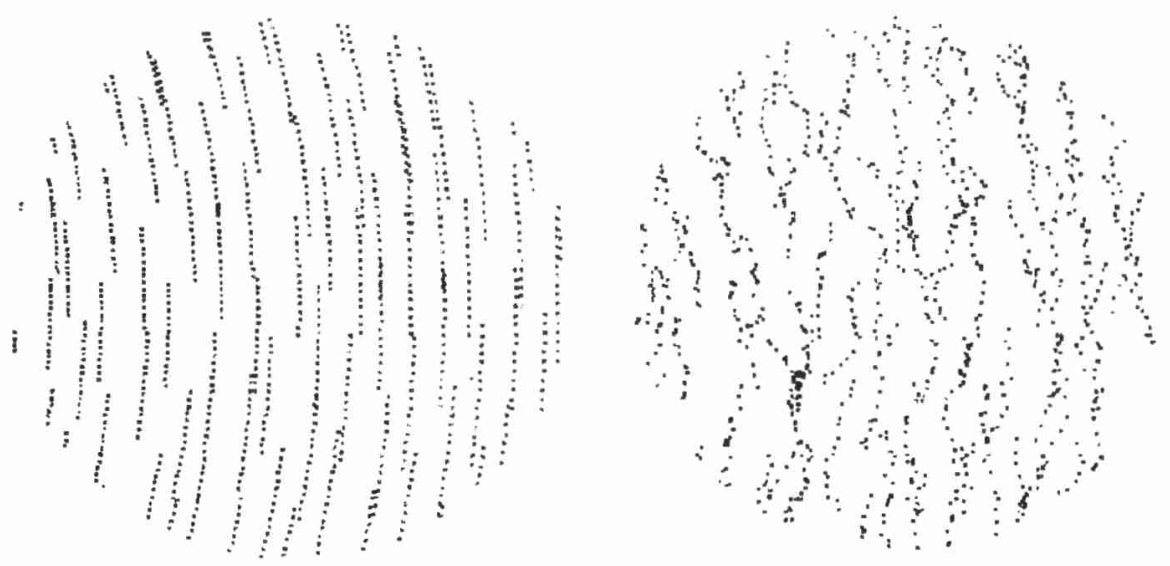

Figure 1. Two examples of the stimulus. The 16 consecutive frames of the stimulus are superimposed. In both cases the experimental parameters are identical: Each frame consists of 64 dots, life time of the dots is 16 frames, vorticity is $1 \mathrm{rad} / \mathrm{s}$ clockwise, and the average translation is $20 \%$ s. Right and left show the conditions with and without the stochastic noise component respectively. 
Procedure. Two female subjects participated in the experiment (two of the authors). $\mathrm{AK}$ is emmetropic; $\mathrm{AD}$ is slightly myopic corrected to normal. Prior to the stimulus onset the subject fixated a fixation cross in the center of the stimulus window. Psychophysical thresholds were measured by disturbing the stimulus configuration with a stochastic noise component (see Figure 1). A 2AFC-paradigm is used to measure the threshold signal to noise ratios as a function of the vorticity and the translation. Under all conditions the task of the subjects was to decide whether the rotation of the stimulus is directed clockwise or anti-clockwise.

\section{Results}

In Figure 2 some of the data of subject AK are shown; the results of subject $\mathrm{AD}$ are similar. The leftmost point of the curves is determined by the minimum translation necessary to locate the center of rotation outside the visible region. The rightmost point, however, gives the maximum translation under which the subject is still able to perform the task. For any higher translation the subject could not even perform the task at the zero noise level! In this figure it can be seen that decreasing the life time of the dots has a clear influence on the curves: Not only the range of measurable points decreases drastically, but also the threshold noise levels and the maximum translation decrease.

The curves in Figure 2 can be characterized by two parameters: a maximum noise level (the rather flat region in each curve) and a maximum translation. In
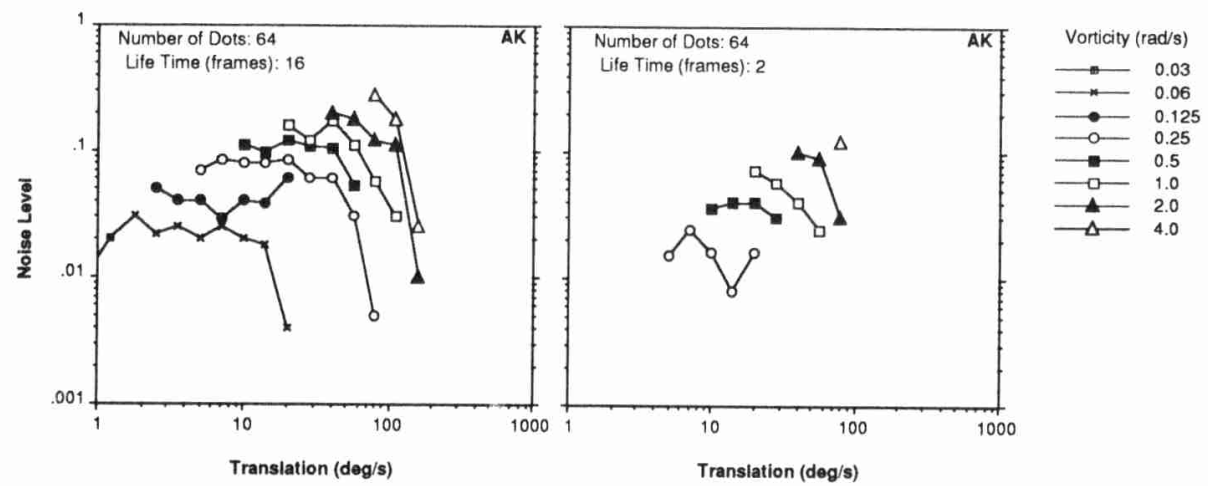

Figure 2. Data of subject AK using 64 dots with a life time of 16 (left) or 2 (right) frames. The points in the graphs reflect all the conditions under which the subject is able to perform the task. 
Figure 3 those two parameters, extracted from the raw data, are shown as a function of the vorticity. In this figure and similar ones the following features appear: (1) the maximum noise level decreases if either the life time or the number of dots decreases, (2) a decrease in the life time of the dots results in a minor decrease of the maximum translation. (3) the number of dots has no significant influence on the maximum translation, and (4) life time and number of dots together determine the range of measurable conditions.
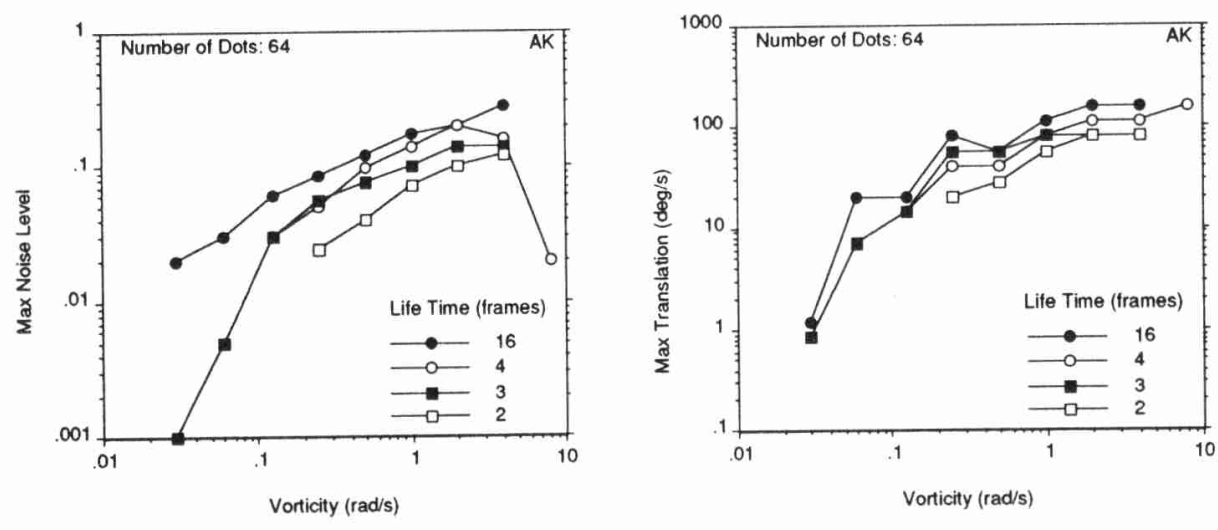

Figure 3. Maximum Noise Level (left) and Maximum Translation (right) as a function of vorticity. Data extracted from curves as in Figure 2.

\section{Discussion}

The experiments should be seen as the first step of a systematic investigation of the capabilities of human observers in perceiving structure in optical flow fields. As such, they cannot in any way be conclusive. Still some points of interest should be emphasized. The detection of vorticity depends critically on the average translational speed. However, Figure 3 shows that the ratio between maximum translation and vorticity is not constant. It follows that the curvature of the flow lines is not the limiting factor of the human performance.

From the results it also becomes clear that the global structure of the stimulus is used at least partially. In those cases where the life time is only two frames, there is locally no information about the curvature of the flow lines. Although performance deteriorates compared to conditions with longer life times, it is still possible to determine thresholds, especially when the number of dots is not too small. 
Acknowledgment. This research is supported by the Insight project of the ESPRIT basic research action of the European Commission.

\section{References}

Gibson, J. J. (1950). The perception of the visual world. Boston: Houghton Mifflin Co.

Koenderink, J. J., \& A. J. Van Doorn (1984). Optical monitoring of ego-motion for movement with respect to a plane surface. In D. Varju \& R. Schnitzler (Eds.), Localization and orientation in biology and engineering. Berlin: Springer Verlag.

Warren, W. H., M. W. Morris, \& M. Kalish (1988). Perception of translational heading from optical flow. Journal of Experimental Psychology: Human Perception and Performance, 14, 646-660. 